\title{
Anesthetic Management of Cesarean Section for Parturient with Takayasu's Arteritis
}

\author{
Youssef Motiaa ${ }^{1,5 *}$, Zakaria Ouassou ${ }^{2,5}$, Khalid Azizi ${ }^{3,5}$ and Wafae El Otmani ${ }^{4,5}$ \\ ${ }^{1}$ Department of Anesthesia, Mokhtar Soussi Hospital, Morocco \\ ${ }^{2}$ Department of Anesthesia, 20 August Hospital, Morocco \\ ${ }^{3}$ Department of Anesthesia, Edderrak Hospital, Morocco \\ ${ }^{4}$ Department of Anesthesiology and Critical Care, Mohamed V Military Hospital of Instruction, Morocco \\ ${ }^{5}$ Department of Anesthesiology and Intensive Care, Ibn Sina University Hospital, Morocco
}

Submission: April 12, 2019; Published: May 17, 2019

*Corresponding author: Youssef Motiaa, Anesthesiology and intensive care department, Ibn Sina University hospital, Faculty of Medicine and Pharmacy, Mohammed V University, Rabat, Morocco

\begin{abstract}
Takayasu Arteritis (TA) is a chronic inflammatory vasculitis of unknown origin affecting aorta and its branches. This vasculitis can cause stenosis, occlusion or aneurysm of the artery, which affects organ perfusion by altering regional circulation. It appears to affect predominantly young women. Pregnancy in women with TA does not affect the inflammatory evolution of the disease but increases the risk of exacerbation of a preexisting chronic hypertension especially in women with advanced cardiovascular abnormalities prior to pregnancy due to physiological hemodynamic changes. Vaginal delivery is possible in this population, but cesarean section can be indicated sometimes for non-obstetric reasons due to the effects of the disease. Because of the diversity and variable degrees of organ dysfunction, anesthesia has special implications and the choice of the anesthetic technique is not standardized. We report two cases of parturient with TA in which emergency and elective cesarean sections were practiced under spinal anesthesia and we discuss the anesthetic implications of cesarean section in parturient with TA.
\end{abstract}

Keywords: Takayasu arteritis; Pregnancy; Cesarean section; Spinal anesthesia

\section{Introduction}

Takayasu Arteritis (TA), also known as "pulseless disease" is a chronic, granulomatous, large-vessel pan-arteritis affecting preferentially the aorta, its major branches (sub-clavian artery, carotid artery and renal arteries) and the pulmonary artery. The changes that occur in vascular layers can result in stenosis, occlusion or aneurysm [1,2] leading to organ hypo-perfusion and dysfunction. This disease affects predominantly young women.

Anesthetic management of patients with TA has some implications for the perioperative care due to various factors: uncontrolled hypertension and complications of the pan-arteritis. One of the most important factors in the anesthetic management of patients with Takayasu is the maintenance of blood pressure intra-operatively and post-operatively. Thus, pre-anesthetic assessment and control of blood pressure, close monitoring, good positioning of the patient on the operating table and choice of the anesthetic technique play a crucial role for successful accomplishment of surgery and prevention of complications. In the obstetric population, Anesthetic management for cesarean section is even more challenging because of the multi-systemic involvement. We report two cases of successful spinal anesthesia in parturients with TA for emergency and elective cesarean sections.

\section{Case Reports}

Spinal anesthesia was performed on two parturients, using $10 \mathrm{mg}$ of heavy bupivacaine $0.5 \%, 25 \mathrm{mcg}$ of fentanyl and $100 \mathrm{mcg}$ of morphine and complete sensory block was achieved at the level of T12 using a 25-gauge Quincke needle. Prior to anesthetic, patients received $20 \mathrm{ml} / \mathrm{kg}$ of IV preloading, $100 \mathrm{mg}$ of IV hydrocortisone and $2 \mathrm{~g}$ of Cefazoline. Standard monitoring included five-lead electrocardiography, non-invasive blood pressure and pulse oximetry. Patients were positioned with left lateral tilt with a pillow under the head to prevent neck hyperextension that might compromise the carotid blood flow. 


\section{Case 1}

A 29-year-old gravida 3 para 3 parturient was admitted on labor at 38 weeks gestation, her medical history included Takayasu Arteritis diagnosed 3 years back following a right ischemic stroke from which she had fully recovered, she was taking prednisolone $5 \mathrm{mg}$ daily and aspirin $75 \mathrm{mg}$ daily orally. Emergency cesarean section was indicated for cephalopelvic disproportion. The patient weighed $70 \mathrm{~kg}$ and was $1.63 \mathrm{~m}$ tall (body mass index $=26.34 \mathrm{~kg} / \mathrm{m} 2$ ), pre-anesthetic examination revealed a heart rate of $90 /$ minute, blood pressure was recorded as $116 / 70 \mathrm{mmHg}$ in the left arm and $90 / 60$ in the right arm, in the right and left lower limbs blood pressure was $113 / 72 \mathrm{mmHg}$ and $115 / 69 \mathrm{mmHg}$ in the right and left lower limbs, respectively. She didn't have any neurologic deficits or muscle atrophy. During her pregnancy, she was diagnosed with gestational diabetes mellitus not requiring insulin; hemoglobin $\mathrm{A} 1 \mathrm{c}$ was $6.8 \%$ and she had gestational hypertension at 28 weeks pregnancy for which she was started on methyldopa $250 \mathrm{mg}$ three times a day. Physical examination did not reveal any symptoms of severe hypertension such as visual changes, neurologic symptoms, dyspnea, jaundice, epigastralgia, oliguria or peripheral edema. Laboratory tests showed normal liver function, kidney function and coagulation. Arterial duplex study showed $75 \%$ stenosis of the right carotid artery $25 \%$ stenosis of the right subclavian artery. Vaginal examination revealed the cervix to be $3 \mathrm{~cm}$ dilated cervix and ultrasound estimated fetal birth weight was $4500 \mathrm{~g}$; thus, cesarean section was indicated for fetal macrosomia.

The parturient was admitted to operating theatre and nursed in left lateral position, she was monitored with a fivelead electrocardiography, pulse oximetry and non-invasive blood pressure in the left upper arm every minute until fetal delivery. Venous access was secured by two 18-gauge peripheral venous catheters and spinal anesthesia was performed, at the L2-L3 level using a 25 -gauge spinal needle with $10 \mathrm{mg}$ of heavy bupivacaine, $100 \mathrm{mcg}$ of morphine and $25 \mathrm{mcg}$ of fentanyl.

Preoperatively, blood pressure remained stable; no episodes of hypotension or neurologic symptoms were recorded following anesthesia and during the procedure. The capillary blood glucose was $0.95 \mathrm{~g} / \mathrm{l}$ before delivery. A $4150 \mathrm{~g}$ live male infant was delivered with an APGAR score of $10 / 10$ at $1 \mathrm{~min}$, a capillary glucose of $0.7 \mathrm{~g} / \mathrm{l}$ and $0.85 \mathrm{~g} / \mathrm{l}$ at birth and 2 hours later respectively. The parturient was the recovery room for $2 \mathrm{~h}$ where she remained stable then to the obstetric unit, she was discharged from the hospital 5 days later.

\section{Case 2}

A 28-year-old woman multigravida was scheduled for elective cesarean section at 37 weeks gestation for cephalopelvic disproportion due to small pelvis. Her past medical history included TA diagnosed 4 years earlier and complicated by transient ischemic attack and optic atrophy, one abortion, two previous cesarean sections, hypertension which was controlled with amlodipine $10 \mathrm{mg}$ once daily. She was also taking aspirin $75 \mathrm{mg}$, prednisone $5 \mathrm{mg}$ and omeprazole $20 \mathrm{mg}$ daily.

Pre-anesthetic evaluation for elective cesarean section revealed a parturient that weighed $65 \mathrm{Kg}$ and was $1.65 \mathrm{~m}$ tall (body mass index $23.87 \mathrm{Kg} / \mathrm{m} 2$ ), she had no neurologic or cognitive abnormalities, pulses were absents in the right upper limb with bilateral carotid bruit; she denied any previous syncopal episodes. Blood pressure was recorded as $122 / 78 \mathrm{mmHg}$ in the left upper arm and $105 / 63 \mathrm{mmhg}$ in the right one. Clinical examination was normal and did not reveal any symptoms of severe gestational hypertension and laboratory tests were normal. She had no predictive criteria of difficult intubation. There were no predicting criteria of difficult intubation and she has a good functional capacity. Arterial doppler ultrasonography revealed anomalous brachiocephalic trunk, $65 \%$ stenosis of the left common carotid artery, $50 \%$ stenosis of the right common carotid artery. The renal ultrasonography Doppler showed a right renal stenosis. Echocardiography showed normal left ventricular function and no valvular or aortic abnormalities. Obstetric ultrasonography did not reveal intrauterine growth restriction.

Venous access was secured in the left upper arm and the patient received $500 \mathrm{ml}$ of saline $0.9 \%$ and IV hydrocortisone $100 \mathrm{mg}$, spinal anesthesia was performed using $10 \mathrm{mg}$ of heavy bupivacaine $0.5 \%, 100 \mathrm{mcg}$ of morphine and $25 \mathrm{mcg}$ of fentanyl via 25-gauge Quincke spinal needle at L2-L3 space. A pillow was placed under her head and $15^{\circ}$ left lateral tilt was maintained by applying a wedge. 5 minutes later, the patient dropped her blood pressure to $80 / 50 \mathrm{mmHg}$, the position was changed to Trendelemburg and $6 \mathrm{mg}$ of ephedrine were administred along with cristalloids with good effect. A $3300 \mathrm{~g}$ live male infant was delivered with an Apgar score of 10 at $1 \mathrm{~min}, 10 \mathrm{IU}$ of oxytocin was infused post-delivery. She was then admitted to the recovery room where she remained stable and she was transferred to the obstetric unit. Regular medication was resumed on the postpartum first day and the patient was discharged home on her third post-operative day.

\section{Discussion}

Anesthetic care of parturients with TA for cesarean section has not been standardized. Clinical cases of cesarean section in TA have been reported, both general anesthesia and regional anesthesia have been described: epidural anesthesia [3-8], combined spinalepidural anesthesia [9] and spinal anesthesia [10-13]. TA is a chronic granulomatous large-vessel vasculitis affecting the aorta and its main branches, especially of the subclavian, common, and internal carotid arteries. The affected vessels are characterized by stenosis, occlusion and sometimes aneurysm. Other vessels can be involved including pulmonary and coronary arteries. The luminal narrowing is due to intimal infiltration by lymphocytes and other inflammatory cells $[1,2,12]$. The aetiology of TA remains unknown but it's suspected to have several causing factors. It affects mainly women of childbearing age in almost $80 \%$ of cases 
[7], thus management of pregnancy and labor is inevitable in this population. Pregnancy does not change the evolution of TA but increases the risk of cardiovascular events due decompensation of hypertension (heart failure, cerebrovascular events) [14].

Comarmond et al. [15] reported in a recent study that the disease activity during pregnancy increases the risk of obstetric complications such as preeclampsia/eclampsia, premature delivery, Intrauterine growth restriction/death and maternal complications; all complicating uncontrolled hypertension and they suggested that it is imperative to control the disease activity and the hypertension before conception and during pregnancy to improve maternal and fetal outcomes [15]. Ishikawa graded TA depending on the presence of four major complications: hypertension, retinopathy, aneurysm formation, and aortic regurgitation [16]. In stage I, none of these complications are present, stage IIa patients have only one of these complications in a mild form, stage IIb patients have only one of these complications but a rather severe one and stage III where more than one complication was present. Ishikawa clinical classification of TA has prognostic and obstetric implications: for non-obstetrical indications, cesarean section is preferred for groups IIb and III because of the risk of cardiac decompensation due to the increase of blood volume during pregnancy and uterine contraction, and it's indicated in groups I and II if the blood pressure is persistently high despite adequate medical therapy. In patients of groups I and IIa, vaginal delivery could be indicated even with hypertension providing that adequate analgesia is ensured $[6,14]$.

Anesthetic management of parturient with TA requires multiple considerations:

a) Hypertension: most frequent and major complication of TA, it can be caused by an abnormal function of the carotid and aortic sinuses and baroreceptors and marked narrowing of aorta and major arteries, but the major cause is renovascular [12]. Severe uncontrolled hypertension complicating TA can lead to end organ damage, stenosis of major blood vessels compromising the regional circulation and difficulties in monitoring arterial blood pressure. Preeclampsia is also fréquent in this population [15].

b) In the nervous system, carotid arteries can be affected and cause serious complications by reducing oxygen flow to the brain such as cerebrovascular accidents. Therefore, it is important to maintain the cerebral perfusion pressure and the cerebral blood flow during anesthesia and surgery.

c) Obstructive changes can also involve coronary arteries exposing to perioperative myocardial infraction, thus an adequate pre-anesthetic evaluation, monitoring and ensuring hemodynamic stability perioperatively and prevention of stress response are important for appropriate anesthetic care.

d) Pulmonary hypertension is the consequence of pulmonary artery involvement and it requires avoiding all factors that increase the pulmonary vascular resistance such as hypoxia, acidemia and hypercapnia.

e) Renal artery stenosis is frequent and is the direct cause if hypertension in TA, therefore perioperative renal protection strategies must be considered during anesthesia [5,10,12]. Angioplasty and vascular stenting or surgery for renal artery stenosis may be necessary if hypertension is uncontrolled despite the medical therapy.

f) For all these reasons, maintaining hemodynamic stability during anesthesia in parturient with $\mathrm{TA}$ is an important anesthetic goal regardless of the anesthetic technique provided [12]. Some authors suggested the use of intra operative Doppler to choose the site for blood pressure monitoring in the absence of prior angiography [8]. For our parturients, standard monitoring included non-invasive blood pressure in the non affected arm where pulse was present and five-lead electrocardiography to detect ischemic abnormalities.

g) TA can be associated to ankylosing spondylitis and rheumatoid arthritis, causing limited joint mobility leading to difficulties in intubation. Airway management requires careful evaluation, the head and neck need to be carefully positioned during anesthesia and the postoperative period [5].

h) Endocrinal repercussion: most parturient with TA receive long-term corticosteroid therapy and are exposed to adrenal insufficiency; perioperative steroid replacement should be warranted to prevent it [5].

i) Another part of anesthetic management of parturient with TA is perioperative drug management; both our patients received steroid replacement to prevent adrenal insufficiency, aspirin and antihypertensive medication (amlodipine and methyldopa) were continued perioperatively. None of our patients was on immunosuppressive therapy.

Indications of cesarean section were obstetrical; the choice of the anesthetic technique is not standardized in the literature; both general and regional anesthesia have been used successfully for this surgery. Regardless of anesthetic technique; the primary objective is to ensure hemodynamic stability in the intraoperative and postoperative periods $[3,12]$.

Neuroaxial anesthesia compared to General Anesthesia (GA) offers some advantages: avoiding the risks associated to GA especially airway management, aspiration and hypertension at critical moments of anesthesia: induction, intubation and extubation. The hypertensive response caused by sympathetic response increases the risk of cardiac failure, cerebral hemorrhage and myocardial infarction $[6,10,12]$. Also, regional anesthesia allows for the best monitoring of cerebral function via the neurological status of an awake patient; Xiao et al. [4] suggested using regional cerebral oxygen saturation as a marker of cerebral hypoperfusion to improve the monitoring of cerebral function during anesthesia for cesarean delivery in a parturient with TA 
to prevent maternal complications despite the use of epidural anesthesia [4]. However, during regional anesthesia, peripheral vasodilation and sympathetic-blockade related hypotension may compromise the regional circulation and jeopardise organ perfusion in patients with TA and vascular abnormalities $[5,10,13]$. To prevent hypotension, most authors reported using epidural anesthesia alone [3-8] or combined to spinal anesthesia [9] to titrate the block level and reduce the risk of hypotension. Both Spinal Anesthesia (SA) and Epidural Anesthesia (EA) provide adequate anesthesia for cesarean section, SA has a shorter onset time but requires more treatment for hypotension [17].

For our patient's pre-anesthetic evaluation did not reveal any symptoms of cardiac failure or claudication although past medical histories included ischemic stroke and transient ischemic attack in the first and second parturients respectively. Both had a good functional status and hypertension was controlled with amlodipine and methyldopa.

Indication for cesarean section was cephalopelvic disproportion in the first case; she was admitted on labor and required rapid delivery. In the second case cesarean was scheduled and spinal anesthesia was provided, however the patient had an episode of hypotension that required a bolus of ephedrine along with intravenous fluids, an epidural could also have been performed and would have provided a better hemodynamic stability. Although the patient didn't develop any neurological complication or kidney injury, rapid changes of blood pressure may have exposed her to a higher risk of kidney injury and cerebral hypoperfusion.

In our unit, we are currently developing a local protocol for anaesthesia in patients with $\mathrm{TA}$, it includes invasive monitoring of blood pressure, the use of opioids in rapid sequence induction when general anaesthesia is indicated and either a combined spinal epidural or epidural anaesthesia when neuraxial anaesthesia is decided.

\section{Conclusion}

Spinal anesthesia for cesarean section in parturients with Takaysu arteritis is a safe technique providing exhaustive preanesthetic evaluation and a close monitoring of neurological status and blood pressure. The most important goal is to avoid hemodynamic changes and to maintain organ perfusion during the perioperative period.

\section{References}

1. Kim ESH, Beckman J (2018) Takayasu arteritis: challenges in diagnosis and management. Heart 104(7): 558-565.
2. Keser G, Aksu K, Direskeneli H (2018) Takayasu arteritis: an update. Turk J Med Sci 48(4): 681-697.

3. Ioscovich A, Gislason R, Fadeev A, Grisaru Granovsky S, Halpern S (2008) Peripartum anesthetic management of patients with Takayasu's arteritis: case series and review. Int J Obstet Anesth 17(4): 358-364.

4. Xiao W, Wang T, Fu W, Wang F, Zhao L (2016) Regional cerebral oxygen saturation guided cerebral protection in a parturient with Takayasu's arteritis undergoing cesarean section: a case report. J Clin Anesth 33: 168-172.

5. Tiwari AK, Tomar GS, Chadha M, Kapoor MC (2011) Takayasu's arteritis: Anesthetic significance and management of a patient for cesarean section using the epidural volume extension technique. Anesth Essays Res 5(1): 98-101.

6. Leal Pda C, Silveira FF, Sadatsune EJ, Clivatti J, Yamashita AM (2011) Takayasus's arteritis in pregnancy. Case report and literature review. Rev Bras Anestesiol 61(4): 479-485.

7. Kuczkowski KM, Fernández CL (2011) Takayasu's arteritis in pregnancy and obstetric anesthesia. J Matern Fetal Neonatal Med 24(10): 1305.

8. Narasimha PK, Chaudhuri S, Joseph TT (2013) Utility of intra-operative ultrasound in choosing the appropriate site for blood pressure monitoring in Takayasu's arteritis. Indian J Anaesth 57(1): 66-68.

9. Ogata J, Horishita T, Shiraishi M, Minami K (2007) Combined spinalepidural anesthesia for cesarean section in a patient with Takayasu arteritis complicated by heart failure. J Anesth 21(4): 525-526.

10. Kathirvel S, Chavan S, Arya VK, Rehman I, Babu V, et al. (2001) Anesthetic management of patients with Takayasu's arteritis: a case series and review. Anesth Analg 93(1): 60-65.

11. Kraemer B, Abele H, Hahn M, Rajab T, Kraemer E, et al. (2008) A successful pregnancy in a patient with Takayasu's arteritis. Hypertens Pregnancy 27(3): 247-252.

12. Gautam S, Srivastava VK, Kumar S, Wahal R (2013) Successful low-dose spinal anesthesia for lower segment caesarean section in a patient with Takayasu arteritis. BMJ Case Rep.

13. Khan AM, Kapoor MC, Puri A (2015) Cesarean section under spinal anesthesia in a patient of Takayasu's arteritis. J Obstet Anaesth Crit Care 5(2): 90-92.

14. Ishikawa K, Matsuura S (1982) Occlusive thromboaortopathy and pregnancy: clinical course and management of 33 pregnancies and deliveries. Am J Cardiol 50(6): 1293-3000.

15. Comarmond C, Mirault T, Biard L, Nizard J, Lambert M, et al. (2015) Takayasu Arteritis and Pregnancy. Arthritis Rheumatol 67(12): 32623269.

16. Ishikawa K (1978) Natural history and classification of occlusive thromboaortopathy (Takayasu's disease). Circulation 57(1): 27-35.

17. Ng K, Parsons J, Cyna AM, Middleton P (2004) Spinal versus epidural anaesthesia for caesarean section. Cochrane Database Syst Rev (2): CD003765. 
CC (i) This work is licensed under Creative Commons Attribution 4.0 License

DOI: 10.19080/JAICM.2019.09.555751

\section{Your next submission with Juniper Publishers} will reach you the below assets

- Quality Editorial service

- Swift Peer Review

- Reprints availability

- E-prints Service

- Manuscript Podcast for convenient understanding

- Global attainment for your research

- Manuscript accessibility in different formats

( Pdf, E-pub, Full Text, Audio)

- Unceasing customer service

Track the below URL for one-step submission https://juniperpublishers.com/online-submission.php 